\title{
Outpatient Morning Report
}

\section{A New Conference for Internal Medicine Residency Programs}

\author{
Anderson Spickard, III, MD, MS, Sean P. Ryan, MD, James Anthony Muldowney, III, MD, \\ Lisa Farnham, MD
}

\begin{abstract}
To clarify the use of outpatient morning report in internal medicine residency programs, we conducted a national survey of internal medicine residency directors and a local survey of a cohort of residents at a large teaching hospital. The program directors reported a $24 \%$ prevalence of outpatient morning report. The cohort of residents reported that the conference contributed much to their education by meeting specific learning needs and covering topics not covered elsewhere in their residency training.
\end{abstract}

KEY WORDS: internship and residency; teaching; internal medicine; morning report.

J GEN INTERN MED 2000;15:822-824.

W ith the shift of internal medicine training to ambulatory teaching sites, many residency programs have adopted a new medical educational venue that reflects the realities of current medical practice: outpatient morning report. Defined as a conference for residents and medical students that is dedicated to the presentation and discussion of outpatient cases, outpatient morning report has many attractive features: it can serve as a locus to execute an outpatient curriculum through casebased teaching, provide opportunities to assess and guide the performances of the participants who present and discuss the cases, and meet the socialization needs of residents and students who gather together after seeing patients at distant clinic sites in order to stimulate and educate one another. Outpatient morning report can complement learning on inpatient experiences by exposing trainees to common outpatient medical problems, the natural history of disease, and curricular items such as medical economics, evidence based medicine, and interviewing skills, which are less commonly covered during inpatient morning report.

Not much is known about outpatient morning report. Whereas national surveys, ${ }^{1,2}$ descriptive studies, ${ }^{3-7}$ and editorials $^{8,9}$ have elucidated the educational and cultural

Received from the Division of General Internal Medicine, Department of Medicine, Vanderbilt University School of Medicine, Nashville, Tenn (AS, SPR, JAM, LF).

Presented at the Annual Meeting, Society of General Internal Medicine, San Francisco, Calif, April 30, 1999.

Address correspondence and reprint requests to Dr. Spickard: Suite 1, Medical Center East, Vanderbilt School of Medicine, Nashville, TN 37232 (e-mail: anderson.spickard-iii@mcmail. vanderbilt.edu). value, as well as the shortcomings, of inpatient morning report, we found only 1 study regarding outpatient morning report. ${ }^{10}$ Malone and Jackson ${ }^{10}$ compared the educational characteristics of inpatient and outpatient morning report at their institution. They found that residents read about and discussed the pathophysiology, differential diagnosis, and management of their patients' problems equally for inpatient and outpatient morning reports, but that outpatient morning report afforded residents a more learner centered setting and more discussion of general internal medicine topics, patient follow-up, and socioeconomic issues than did inpatient morning report.

To determine how many internal medicine residency programs use outpatient morning report, how it is used, and how it is valued, we conducted 2 studies: (1) a national survey of internal medicine residency program directors about the use of outpatient morning report, and (2) a local survey involving a 1-year prospective evaluation of the perceptions of residents who participated in outpatient report at Vanderbilt University, a large teaching institution.

\section{METHODS}

\section{Study Design}

The prevalence of outpatient morning report was determined from a brief questionnaire that was sent to the residency program directors of 404 internal medicine departments who were identified through the 1998-1999 Graduate Medical Education Directory. Respondents were asked whether their program has an outpatient morning report; if so, they were asked to describe the format including how often it occurs, who attends the sessions, who leads the discussions, and who chooses and presents the cases. Outpatient morning report was defined for the program directors as a "conference for residents and/or students, separate from inpatient morning report, where outpatient cases are presented and discussed." Pre- or postclinic conferences, journal clubs, or inpatient morning reports that included only an occasional discussion of an outpatient case were not considered in the definition. The report had to be offered to all residents in the program at the same time. If the program replaced inpatient morning report with a conference devoted to the presentation of outpatient cases at least once a month, it qualified as an outpatient morning report. All program directors who responded affirmatively to having an outpatient morning report were contacted by telephone to confirm that the conference met the above criteria. 
To understand the impact of outpatient morning report at a large teaching institution, we surveyed 77 internal medicine residents at Vanderbilt University who participated in outpatient morning report over the course of 1 year. This conference has been described elsewhere. ${ }^{11}$ The format includes case presentations by residents and medical students followed by interactive discussions led by faculty members or chief residents. At the end of the session, the presenter provides a handout and a 5-minute discussion about a topic related to the case. ${ }^{11}$ The outcome measure in this study included a 10-item survey instrument derived from a 44-item survey of inpatient morning report ${ }^{7}$ and from the observations by three of the authors (AS, SR, LF) who had a collective experience of 12 years of participation in outpatient morning report. On a 5-point scale, residents were asked to rate the educational value of outpatient morning report, the learning atmosphere, the leadership skills of the chief residents, the practicality of the cases, and the ability of the conference to meet personal learning needs and to cover topics not covered elsewhere in their training. Furthermore, residents were asked whether the presence of students "added to," "detracted from," or "did not impact" their learning at the conference.

\section{Statistical Analysis}

Descriptive statistics were calculated for the results from each survey. Fisher's exact test was used to compare the size of the residency programs and use of outpatient morning report. All analyses were performed using SAS software (SAS Institute, Cary, NC, 1989).

\section{RESULTS}

\section{National Survey of the Use of Outpatient Morning Report}

Three hundred seventy (92\%) of 404 internal medicine residency program directors responded to the survey. Eighty-eight programs (24\%) had an outpatient morning report. Many residency directors of programs without an outpatient morning report were enthusiastic about the idea of starting one (data not shown). Residency programs with 50 or more residents ( $n=166$ programs) were more likely to have an outpatient morning report than programs with less than 50 residents ( $n=204$ programs) (37\% vs $15 \% ; P<.001$. The characteristics of outpatient morning reports used by U.S. internal medicine programs are shown in Table 1. Almost half of the programs conducted their reports more than once a week. In general, attending physicians or chief residents led the conference, while residents typically chose and presented the cases. One fourth of the chief medical residents who attended outpatient morning report were designated by program directors as "outpatient chief residents." Although many programs invited medical students to attend outpatient morning report, only a small percentage of them allowed students to choose or present patient cases.

\section{Local Survey at Vanderbilt University}

Ratings of the Conference. Over the course of the year, 64 (83\%) of 77 residents responded to the outpatient morning report survey. The residents rated the educational value of outpatient morning report 4.7 on a 5-point Likert scale. Over $88 \%$ of the residents rated features of the conference as very good or outstanding (equivalent to a 4 or 5 on the 5-point Likert scale), including the learning atmosphere, the leadership skills of the chief residents, the practicality of the cases, and the ability of the conference to meet personal learning needs and to cover topics not covered elsewhere in their training. Forty percent of the residents thought that the presence of the medical students added to their learning at morning report; none of them thought that students detracted from their learning.

Table 1. Characteristics of Outpatient Morning Report in U.S. Internal Medicine Residency Programs

\begin{tabular}{lc}
\hline \hline & $n(\%)$ \\
\hline Programs with outpatient morning report & $88(23.8 \%)$ \\
Frequency of sessions & $12(13 \%)$ \\
1-2 times/month & $35(40 \%)$ \\
1 time/week & $41(47 \%)$ \\
2-5 times/week & \\
Who attends the sessions?* & $82(93 \%)$ \\
Attending physician & $59(67 \%)$ \\
Chief resident & $88(100 \%)$ \\
Resident & $58(66 \%)$ \\
Medical student & \\
Who leads the sessions?* & \\
Attending physician & $53(60 \%)$ \\
Chief resident & $40(45 \%)$ \\
Resident & $24(27 \%)$ \\
Medical student & $1(1 \%)$ \\
Who chooses the cases?* & \\
Attending physician & $32(36 \%)$ \\
Chief resident & $32(36 \%)$ \\
Resident & $64(73 \%)$ \\
Medical student & $10(11 \%)$ \\
Who presents the cases?* & \\
Attending physician & $20(23 \%)$ \\
Chief resident & $13(15 \%)$ \\
Resident & $85(97 \%)$ \\
Medical student & $17(19 \%)$ \\
\hline
\end{tabular}

* Respondents could check as many options as applied for this category. Therefore, the sum of the percentages for this category exceeds $100 \%$.

${ }^{\dagger}$ Twenty percent of medical students were classified as third-year students, $18 \%$ as fourth-year students, $62 \%$ as third- and fourthyear students. 


\section{DISCUSSION}

Approximately one fourth of internal medicine residency programs have an outpatient morning report, as defined in this study. This may be a surprisingly high prevalence to the reader who has not heard of outpatient morning report and who wonders about the proof of its value and the resources needed to conduct the conference. It may be a surprisingly low prevalence to the reader who considers the current curricular emphasis on outpatient medicine to be inadequate and who considers the definition of outpatient morning report in this study to be too lenient.

It is perhaps not surprising to anyone that the general format of outpatient morning report used across the country appears to be quite similar to inpatient morning report. Residents present cases and faculty members and chief residents discuss them. Students join the session in $66 \%$ of the programs but have a participatory role in choosing or presenting cases in less than $20 \%$ of the programs.

The local study results at Vanderbilt show that residents highly valued outpatient morning report. Residents reported that specific educational needs were met during this conference. This may reflect the supportive atmosphere of the conference and the exposure to practical cases not covered elsewhere in their residency. They found chief residents to be superb leaders and teachers of outpatient morning report. In contrast to studies of inpatient morning report, ${ }^{7}$ residents at Vanderbilt were positive about students participating in outpatient morning report. Many residents (40\%) felt that the contributions from students at morning report added to their learning. This may be explained by the active role of students (who were required to present cases and provide handouts), the interactive format, and less disparity between learning levels of the participants as the students who participate in outpatient morning report at Vanderbilt are in their senior year of training.
Limitations must be considered in the interpretation of the results of these studies. The national study results are limited by the definition of outpatient morning report and by the reliance on the self-reports of residency program directors. The local study results are limited by the measurement of residents' attitudes at a single program. Measurement of the impact of outpatient morning report on the knowledge and behaviors of residents, and ultimately patient outcomes, at different training sites would be appropriate endpoints for future studies. Future articles should explore the educational value of varied formats and content of outpatient morning report and delineate barriers and solutions to starting such conferences.

The authors would like to thank James W. Pichert, PhD for his editorial assistance.

\section{REFERENCES}

1. Parrino TA, Villanueva AG. The principles and practice of morning report. JAMA. 1986;256:730-3.

2. Schiffman FJ, Mayo-Smith MF, Burton MD. Resident report: a conference with many uses. R I Med. 1990;73:95-102.

3. Hill RF, Tyson EP, Riley HD. The culture of morning report: ethnography of a clinical teaching conference. South Med J. 1997;90:594-600.

4. Mehler PS, Kaehny WD, Fraser V, et al. Clinical effect of morning report. Acad Med. 1993;68:547.

5. Ramratnam B, Kelly G, Mega A, Tilkemeier P, Schiffman FJ. Determinants of case selection at morning report. J Gen Intern Med. 1997;12:263-6.

6. Reilly B, Lemon M. Evidence-based morning report: a popular new format in a large teaching hospital. Amer J Med. 1997;103:419-26.

7. Ways M, Kroenke K, Umali J, Buchwald D. Morning report: a survey of resident attitudes. Arch Intern Med. 1995;155:1433-6.

8. Parrino TA. The social transformation of medical morning report. J Gen Intern Med. 1997;2:332-3.

9. Brancati FL. Morning distort. JAMA. 1991;266:1627.

10. Malone ML, Jackson TC. Educational characteristics of ambulatory morning report. J Gen Intern Med. 1993;8:512-4.

11. Spickard A III, Hales JB, Ellis S. Outpatient morning report: a new educational venue. Acad Med. 2000;75:297. 